\title{
Relação entre estressores, estresse e ansiedade
}

\author{
Regina Margis* \\ Patrícia Picon** \\ Annelise Formel Cosner** \\ Ricardo de Oliveira Silveira ${ }^{* * *}$
}

\section{INTRODUÇÃO}

O termo estresse denota o estado gerado pela percepção de estímulos que provocam excitação emocional e, ao perturbarem a homeostasia, disparam um processo de adaptação caracterizado, entre outras alterações, pelo aumento de secreção de adrenalina produzindo diversas manifestações sistêmicas, com distúrbios fisiológico e psicológico. O termo estressor por sua vez define o evento ou estímulo que provoca ou conduz ao estresse. ${ }^{1}$

Em 1936 o fisiologista canadense Hans Selye introduziu o termo "stress" no campo da saúde para designar a resposta geral e inespecífica do organismo a um estressor ou a uma situação estressante. Posteriormente, o termo passou a ser utilizado tanto para designar esta

\footnotetext{
* Psiquiatra, mestranda em Bioquímica (UFRGS).

** Psiquiatra, professora assistente do Departamento de Psiquiatria FAMED-PUCRS.

*** Psiquiatra, psiquiatra do Ambulatório de Transtornos de Ansiedade do Hospital São Lucas.

${ }^{* * * *}$ Psiquiatra, mestrando em Psiquiatria (UFRGS) e membro do corpo clínico do Prontopsiquiatria - CISAME.
}

resposta do organismo como a situação que desencadeia os efeitos desta., ${ }^{1,2}$

A resposta ao estresse é resultado da interação entre as características da pessoa e as demandas do meio, ou seja, as discrepâncias entre o meio externo e interno e a percepção do indivíduo quanto a sua capacidade de resposta. Esta resposta ao estressor compreende aspectos cognitivos, comportamentais e fisiológicos, visando a propiciar uma melhor percepção da situação e de suas demandas, assim como um processamento mais rápido da informação disponível, possibilitando uma busca de soluções, selecionando condutas adequadas e preparando o organismo para agir de maneira rápida e vigorosa. A sobreposição destes três níveis (fisiológico, cognitivo e comportamental) é eficaz até certo limite, o qual uma vez ultrapassado, poderá desencadear um efeito desorganizador ${ }^{2}$. Assim, diferentes situações estressoras ocorrem ao longo dos anos, e as respostas a elas variam entre os indivíduos na sua forma de apresentação, podendo ocorrer manifestações psicopatológicas diversas como sintomas inespecíficos de depressão ou ansiedade, ou transtornos psiquiátricos definidos, como por exem-

Recebido em 24/01/2003. Revisado em 11/03/2003. Aprovado em 18/03/2003. 
plo o Transtorno de Estresse Pós-Traumático3.

O presente trabalho tem por objetivo relatar os achados de literatura reunidos por grupo de estudos do XVI Ciclo de Avanços em Clínica Psiquiátrica, a ser realizado em 13 de abril de 2003, em Porto Alegre. O foco de interesse foi o estudo da relação entre estresse, eventos de vida estressores e sintomas de ansiedade. Não sendo propósito do grupo estudar a relação entre eventos traumáticos e transtornos já descritos e categorizados segundo a Classificação Internacional de Doenças (CID - 10) ${ }^{3}$, como o transtorno de estresse pós-traumático, reação aguda ao estresse, reação de ajustamento ao estresse e alteração de personalidade após experiência catastrófica. O grupo utilizou o sistema de busca on-line nas bases de dados MEDLINE e LILACS, no período de 1994 a janeiro de 2003. Os termos de busca foram life-events, stress, anxiety. Cabe salientar a relativa escassez de trabalhos localizados na área de interesse.

\section{ESTRESSORES}

As situações ambientais podem ser provocadoras de estresse e agrupadas como: acontecimentos vitais (life-events), acontecimentos diários menores e situações de tensão crônica. Os chamados life-events, estudados pela primeira vez por Holmes e Rahe em $1967^{2}$, têm sido um grande foco da epidemiologia psiquiátrica nas últimas décadas. Na literatura, os lifeevents têm sido nomeados como acontecimentos vitais, eventos de vida, eventos estressores ou eventos de vida negativos. Os autores propõem que a nomenclatura mais apropriada para a discussão do tema seja a de eventos de vida estressores, a qual será utilizada no decorrer deste artigo. Os eventos de vida estressores têm sido diferenciados em dependentes e independentes. Os dependentes apresentam a participação do sujeito, ou seja, dependem da forma como o sujeito se coloca nas relações interpessoais, como se relaciona com o meio, onde seu comportamento provoca situações desfavoráveis para si mesmo. Os eventos de vida estressores independentes são aqueles que estão além do controle do sujeito, independem de sua participação, sendo inevitáveis, como por exemplo, a morte de um familiar ou a saída de um filho de casa como parte do ciclo vital de desenvolvimento. ${ }^{4}$

Há de se fazer ainda distinção entre evento traumático e evento de vida estressor. O evento traumático é aquele em que, uma vez a ele psíquicas por um tempo longo, podendo chegar a décadas, mesmo após seu afastamento do mesmo. O evento traumático grave inclui aspectos relacionados ao comprometimento da integridade física do próprio indivíduo ou de outrem. O evento de vida estressor, por outro lado, é aquele que, embora possa dar origem a efeitos psicológicos sob a forma de sintomas e desadaptação, uma vez removido, tende a acarretar uma diminuição do quadro psicopatológico por ele provocado. ${ }^{5}$

Mudanças importantes na vida, como iniciar um novo emprego, casar-se ou separar-se, o nascimento de um filho, sofrer um acidente, podem gerar resposta de estresse nos indivíduos a elas expostos. Avaliar a ocorrência destes eventos pode ser uma forma de tomar conhecimento da freqüência com que determinada pessoa desencadeia uma resposta de estresse.

Além dos eventos de vida estressores, os denominados acontecimentos diários menores, que podem ser vivenciados em diversas situações cotidianas, como perder coisas, esperar em filas, ouvir o som do despertador ou o baruIho provocado por vizinhos, também são provocadores de resposta de estresse. Muitas vezes estes acontecimentos diários menores, quando freqüentes, geram resposta de estresse com efeitos psicológicos e biológicos negativos mais importantes do que eventos de vida estressores de menor freqüência. Salienta-se, então, a importância destes eventos menores, porém freqüentes, que para alguns indivíduos são provocadores de grande desconforto psíquico.

O terceiro grupo de situações ambientais provocadoras de estresse corresponde às situações de tensão crônica que geram estresse relativamente intenso e que persistem ao longo do tempo, como por exemplo, um relacionamento conjugal perturbado (com agressões verbais e físicas ao longo de anos), gerando importantes efeitos psicopatológicos. ${ }^{2}$

\section{RESPOSTA AO ESTRESSE}

\subsection{Nível cognitivo}

A resposta ao estresse depende, em grande medida, da forma como o indivíduo filtra e processa a informação e sua avaliação sobre as situações ou estímulos a serem considerados como relevantes, agradáveis, aterrorizantes, etc. Esta avaliação determina o modo de responder diante da situação estressora e a forma como o mesmo será afetado pelo estresse. No nível cognitivo, podemos então distin- 
guir quatro componentes: 1. avaliação inicial automática da situação ou estímulo, também conhecida como reação afetiva, em que o sujeito avalia inicialmente o potencial de ameaça para si. Esta avaliação global afetiva determina um padrão de respostas do tipo defesa ou conferência e orientação. Quando a situação ou estímulo é percebido como ameaçador, então uma resposta de defesa é ativada. Porém, se a avaliação for de não ameaça, a resposta de conferência e orientação é a escolhida, e o sujeito se prepara para recolher mais informações. As respostas de conferência e orientação ou de defesa irão provocar diferentes respostas fisiológicas; 2. avaliação da demanda da situação ou avaliação primária, em que o sujeito avalia a situação estressora, não por seu significado intrínseco, mas de acordo com sua história pessoal e seu aprendizado e experiências prévias. Nesta fase, o relevante é como o sujeito vivencia a situação de estresse; 3 . avaliação das capacidades para lidar com a situação estressora ou avaliação secundária, quando o sujeito avalia a situação em relação às suas capacidades e recursos de enfrentamento para manejá-la e; 4. organização da ação ou seleção da resposta, a partir das avaliações anteriormente descritas, em que o sujeito elabora suas respostas às demandas percebidas ${ }^{2}$.

As respostas podem ser específicas para a situação alvo ou gerais, ou pode ainda não haver resposta disponível para o sujeito que então decidirá entre arriscar uma nova resposta ou suportar passivamente a situação estressora. Os recursos comportamentais e fisiológicos a serem mobilizados dependem, em grande medida, desta escolha.

\subsection{Nível comportamental}

As respostas comportamentais básicas diante de um estressor são: enfrentamento (ataque), evitação (fuga), passividade (colapso). As habilidades do sujeito para dar respostas adequadas a cada estressor dependem de um aprendizado prévio das condutas pertinentes e de se a emissão de respostas recebeu reforço nas situações similares precedentes. Além disto, a resposta de enfrentamento será modulada por suas conseqüências. A resposta de enfrentamento selecionada define a forma de ativação do sujeito, os recursos e estruturas fisiológicas a serem mobilizadas e os possíveis transtornos psicofisiológicos que possam ocorrer. A resposta ao estressor pode ser preditiva de transtornos específicos como no caso de fuga e evitação, ser preditiva de agorafobia ou fobia social, ou um padrão de enfrentamento da personalidade tipo A ser preditor de transtornos cardiovasculares. ${ }^{2}$

\subsection{Nível fisiológico}

\section{Aspectos neuroanatômicos}

Do ponto de vista evolutivo, a ansiedade e o medo, assim como o estresse, têm suas raízes nas reações de defesa dos animais, que ocorrem em resposta aos perigos encontrados em seu meio ambiente. Quando um animal se depara com uma ameaça ao seu bem estar, à sua integridade física, ou até mesmo à sua sobrevivência, ele experimenta uma série de respostas comportamentais e neurovegetativas, que caracterizam a reação de medo (ver fluxograma 1).

Considera-se que diferentes estruturas cerebrais estejam envolvidas nas diferentes estratégias de defesa, dependendo do nível de ameaça percebido pelo indivíduo. Experiências com modelos animais evidenciam que, em situações potencialmente perigosas (situações novas ou situações semelhantes àquelas nas quais o indivíduo viveu um perigo real em um outro momento), as estruturas envolvidas seriam o sistema septo-hipocampal e a amígdala. Tais estruturas recebem informações colhidas pelos diferentes sistemas sensoriais, criando assim uma representação do mundo exterior. $\mathrm{O}$ sistema septo-hipocampal executaria inicialmente a função de conferidor, comparando a síntese dos dados sensoriais do momento, com as predições que levam em conta as memórias armazenadas em diversos locais do Sistema Nervoso Central (SNC), bem como os planos de ação gerados pelo córtex pré-frontal. Quando há coerência entre as duas representações, o sistema septo-hipocampal continuaria a executar sua tarefa de conferidor. Entretanto, quando é detectada uma discrepância entre o esperado e o acontecido, o sistema septo-hipocampal passaria a funcionar na modalidade controle, gerando inibição do comportamento, aumento do nível de vigilância, dirigindo a atenção do indivíduo para possíveis fontes de perigo (comportamento de avaliação de risco).

Quando os sinais de perigo tornam-se explícitos, mas encontram-se ainda à longa distância, a reação típica é a de imobilidade tensa (congelamento ou inibição comportamental defensiva), cujo substrato neural provavelmente seja a porção ventral da matéria cinzenta periaquedutal (MCP) do mesencéfalo.6,7 Graeff e cols., baseados em experimentos com animais, 
consideram que a MCP parece ser a principal estrutura responsável pela programação de luta e fuga, que guarda analogia com os ataques de pânico ${ }^{6}$. Esta, juntamente com o hipotálamo, programa as manifestações comportamentais, hormonais e neurovegetativas das reações de defesa. Além disso, foi também verificado que a estimulação da via serotonérgica, que se origina no núcleo mediano da rafe e inerva preferencialmente o septo-hipocampo, determina inibição comportamental característica da defesa.

\section{Neurotransmissores e estresse}

Diferentes substâncias têm sido estudadas visando a compreender a neurofisiologia que envolve a ansiedade e o estresse. Entre elas as aminas biogênicas, como a noradrenalina, a dopamina e a serotonina; aminoácidos, como o ácido gama-aminobutírico (GABA), a glicina e o glutamato; peptídeos, como o fator de liberação de corticotropina (CRF), o hormônio adrenocorticotrófico $(\mathrm{ACTH})$ e a colecisticinina (CCK) e esteróides, como a corticosterona (fluxograma 2).

No SNC, neurônios que sintetizam noradrenalina estão situados nas regiões bulbar e pontina, sendo que o grupo mais importante situa-se no locus ceruleus. As células do locus ceruleus, quando ativadas por estímulos estressantes, ameaçadores, produzem uma reação comportamental cardiovascular característica de medo. Acredita-se que o locus ceruleus funcione como um "sistema de alarme", ou seja, exerce a função de atenção, monitorando continuamente 0 ambiente e preparando o organismo para situações de emergência. ${ }^{6,7}$

De todos os transtornos de ansiedade, o transtorno do pânico e o estresse pós-traumático são os que apresentam evidências mais contundentes de uma anormalidade do sistema noradrenérgico.

A noradrenalina tem sido relacionada também com experiências intrusivas. O locus ceruleus inerva hipocampo, amigdala e neocortex temporal, que são algumas das estruturas neuroanatômicas envolvidas no processo associativo. Na resposta aguda ao estresse, há um aumento importante de noradrenalina na fenda sináptica, resultando num aumento da resposta monosináptica evocada. ${ }^{8}$

Com relação à dopamina, o estresse aumenta a liberação e o metabolismo deste neurotransmissor no córtex pré-frontal, uma área envolvida na produção de respostas ao estres$\mathrm{se}^{9}$. O envolvimento da dopamina com estados
Sabe-se que a diminuição da função serotonérgica pode resultar em aumento da função da dopamina, promovendo hipervigilância nas situações de estresse.

Acredita-se atualmente que a serotonina exerça um duplo papel na regulação do comportamento de defesa. Os sinais de perigo estimulariam o sistema de defesa através da amígdala e, ao mesmo tempo, ativariam os neurônios serotonérgicos do núcleo dorsal da rafe. Estes, por vias nervosas diferentes, inervam tanto a amígdala quanto a MCP. A serotonina facilitaria as reações ativas de defesa na amígdala e as inibiria na MCP. As respostas mediadas pela serotonina teriam, portanto, um sentido adaptativo, já que para níveis de perigo potencial ou distal é conveniente que os comportamentos de luta e fuga sejam inibidos, possibilitando que 0 indivíduo adote estratégias mais adequadas, como exploração cautelosa e inibição comportamental. ${ }^{7}$ Poder-se-ia dizer, ainda, que a serotonina aumenta a ansiedade atuando na amígdala e contém o pânico agindo na MCP.

O GABA (acido gama-aminobutírico) é o principal neurotransmissor inibitório do Sistema Nervoso Central. No núcleo dorsal da rafe, exerce uma inibição tônica sobre os neurônios serotonérgicos. Na MCP e colículos superiores, exerce um controle inibitório sobre o substrato neural do medo. No teto mesencefálico, controla aspectos motores relacionados ao comportamento de fuga ${ }^{6,7}$.

Os receptores benzodiazepínicos (BZD) e o GABA estão presentes em toda parte no SNC, afetando diversos sistemas funcionais. Entretanto, os sistemas neuronais envolvidos na regulação da ansiedade, segundo evidências experimentais, são particularmente os núcleos lateral e baso-lateral da amígdala, os quais são ricos em receptores BZD. Estudos mostram também que o sistema GABA - BZD da amígdala regula não apenas a ansiedade, mas a memória emocional, já que à amígdala também é atribuída a ação amnésica dos compostos BZD. 6,7

Resumindo, nas situações em que o perigo está próximo, o indivíduo irá reagir com comportamentos vigorosos de luta ou fuga. Para tal, fazem-se necessárias alterações cardiovasculares, constituindo em elevação da pressão arterial, taquicardia, vasoconstrição na pele e nas vísceras e vasodilatação nos músculos estriados, bem como hiperventilação. O sistema neural responsável por tais manifestações comportamentais e neurovegetativas de defesa ativa seria a MCP dorsal e o hipotálamo medial. O hipotálamo, uma vez estimulado, libera o fator 
liberador de corticotrofina (CRF), vasopressina e outros neuropeptídeos reguladores. A liberação de CRF promove, entre outras, a secreção do hormônio adrenocorticotrófico (ACTH), o qual leva à liberação do cortisol pelas adrenais (eixo hipotálamo-hipófise-adrenal) ${ }^{10}$. Níveis de cortisol cronicamente elevados podem interferir na estrutura e função hipocampal, produzindo alterações de memória e cognição ${ }^{11}$.

As situações de estresse produzem, portanto, um aumento geral da ativação do organismo, a fim de que o indivíduo possa reagir. Inicialmente considerava-se que esta ativação fisiológica fosse genérica e indiferenciada para qualquer estressor. Entretanto, hoje se pensa que diferentes mecanismos neurais e endócrinos estão envolvidos na resposta ao estresse e que podem ser ativados seletivamente. Labrador e cols. $(1994)^{2}$ distinguem três eixos de atuação da resposta fisiológica ao estresse:

- O eixo neural, o qual se ativa imediatamente, frente a uma situação de estresse. Implica a ativação principalmente do sistema nervoso autônomo (feixe simpático) e do sistema nervoso periférico. Seus efeitos são: aumento do ritmo cardíaco (SNA), aumento da pressão arterial (SNA), secura na boca (SNA), sudorese intensa (SNA), "nó" na garganta (SNA), formigamento dos membros (SNP), dilatação das pupilas (SNP) e dificuldade para respirar.

- O eixo neuroendócrino é mais lento em sua ativação e necessita de condições de estresse mais duradoras. Seu disparo ativa a medula das suprarrenais, provocando a secreção de catecolaminas (adrenalina e noradrenalina), o que ajuda a aumentar e manter a atividade adrenérgica somática, produzindo efeitos similares aos gerados pela ativação simpática. É o eixo de luta e fuga, pois prepara o organismo para uma intensa atividade muscular, quando a pessoa percebe que pode fazer algo para controlar a situação (seja enfrentar ou fugir). Seus efeitos são: aumento da pressão arterial, do aporte sangüíneo para o cérebro, do ritmo cardíaco, da estimulação dos músculos estriados, de ácidos graxos, triglicerídeos e colesterol no sangue; secreção de opióides endógenos e diminuição do fluxo sangüíneo nos rins, no trato gastrointestinal e na pele. Esta resposta aumenta o risco de hipertensão, de formação de trombos, de angina do peito, em pessoas propensas. Também aumenta o risco de risco de arritmias, elevando a possibilidade de morte súbita.

- O eixo endócrino caracteriza-se por disparo mais lento e por efeitos mais duradouros que os anteriores e necessita de que a situação de estresse mantenha-se por mais tempo. Este eixo é disparado quando a pessoa não dispõe de estratégias de enfrentamento na situação de estresse. Seus principais efeitos são: aumento da glicogênese, aumento da produção de corpos cetônicos, exacerbação de lesão gástrica, aumento da produção de uréia, aumento da liberação de ácidos graxos livres no sistema circulatório, aumento da suscetibilidade a processos ateroscleróticos, aumento da suscetibilidade à necrose miocárdica, supressão de mecanismos imunológicos, diminuição do apetite.

\section{GENÉTICA E AMBIENTE: O PAPEL DOS ESTRESSORES}

O modelo multicausal de transtornos mentais prevê a influência de fatores genéticos e ambientais. Neste modelo, uma relação longitudinal causal entre os eventos estressores, o surgimento de sintomas e de transtornos mentais apresenta plausibilidade biológica aceitável.

Existem indicativos de associação entre a exposição a eventos de vida estressores negativos e depressão. Brown e cols. $(1987)^{12}$, em uma revisão de 10 estudos populacionais com mulheres deprimidas, concluíram que em média $83 \%$ dos casos apresentavam eventos de vida estressores anteriores ao surgimento do quadro depressivo. Entretanto, nem todos os sujeitos expostos desenvolveram psicopatologia, e uma em cinco mulheres expostas a eventos estressores desenvolveram depressão. Achados como este nos remetem para a questão da variabilidade individual.

Segundo Kendler e cols. (1999) ${ }^{13}$ numerosos estudos, em especial no campo da depresão, têm demonstrado que a exposição a eventos de vida estressores é substancialmente influenciada por fatores genéticos. Alguns indivíduos não se expõem a eventos de vida estressores ao acaso, mas apresentam uma tendência para selecionar situações com maior probabilidade em se constituir num evento de vida estressor. Os fatores de risco genéticos para eventos de vida estressores se correlacionam positivamente com os fatores de risco genéticos para depressão maior. Então um conjunto de traços geneticamente influenciados aumenta a probabilidade do indivíduo selecionar para si situações de alto risco ambiental que se constituam em eventos de vida estressores, o que aumenta sua vulnerabilidade para o surgimento de depressão maior.

A avaliação negativa do evento estressor pode ser atribuída a uma vulnerabilidade que 
não está ligada ao evento estressor propriamante dito. A variabilidade individual provavelmente se deva a uma suscetibilidade mediada geneticamente, que influencia a forma do indivíduo avaliar e enfrentar os eventos de vida estressores dependentes e independentes, ou mesmo provocar aqueles dependentes.

Posto que as relações sociais são intrinsecamente diádicas e a forma como o indivíduo se comporta influencia a resposta social que vai obter, o comportamento do indivíduo levará a menor ou maior risco de expor-se a eventos de vida estressores do tipo dependente. A relação causal entre eventos de vida estressores dependentes e depressão tem sido mais estudada, e as evidências são de que entre adolescentes esta relação é significativa. ${ }^{4}$

Apesar dos eventos de vida estressores dependentes estarem mais fortemente relacionados com depressão maior do que eventos independentes, esta relação não parece ser do tipo causal em sua totalidade. Em uma coorte prospectiva do estudo de base populacional do "Virginia Twin Registry", avaliada por Kendler e cols. (1999) ${ }^{13}$, a probabilidade de depressão na presença de evento dependente foi $80 \%$ maior do que na presença de eventos de vida estressores independentes. A proporção de associação causal entre eventos estressores e depressão maior foi de $65 \%$. Desta forma, um conjunto de traços genéticamente determinados, os quais provavelmente se refletem em um "temperamento dificil ou neurótico", predispõem o indivíduo a se expor a eventos de vida estressores e a episódios de depressão maior. Eventos de vida estressores parecem ter, portanto, uma relação causal com a apresentação de depressão maior. Entretanto, um terço da associação entre os eventos de vida estressores e a apresentação de depressão maior não é causal, uma vez que os indivíduos predispostos a episódios depressivos se colocam em situações de maior risco ambiental, ou seja, diante de eventos de vida estressores. ${ }^{12}$

Silberg e cols. (1999) ${ }^{14}$ realizou estudo com gêmeos no "Virginia Twin Registry" para avaliar o desenvolvimento comportamental de adolescentes e investigou a trajetória de sintomas depressivos entre meninos e meninas, da infância até a adolescência. O estudo buscou estimar a associação entre a suscetibilidade genética à depressão e eventos de vida estressores dependentes e independentes, como por exemplo mau desempenho na escola ou brigas com colegas e amigos, no ano que antecede o surgimento do transtorno depressivo. Entre os meninos, não poder participar do time de espor- tes, ou de uma banda de música, e perder um amigo por brigas verbais foram depressogênicos. Entre as meninas, o evento estressor mais depressogênico foi o rompimento com um namorado. O impacto dos eventos de vida estressores foi significativo entre as meninas e meninos, mas foi mais importante entre meninas acima de 12 anos, que possuíam maior taxa de herdabilidade para depressão. Os resultados confirmaram a influência genética na depressão entre adolescentes (taxa de concordância de monozigóticos de 0,37 e dizigóticos de 0,09) e a influência ambiental dos eventos de vida estressores (taxa de concordância entre monozigóticos de 0,91 e dizigóticos de 0,75). A taxa de herdabilidade para depressão neste grupo foi estimada em $30 \%$ e o restante foi devido a fatores ambientais, entre os quais os eventos estressores. Os eventos estressores negativos tiveram um papel etiológico mais preponderante entre os meninos; nas meninas, o papel da herança genética foi maior, bem como a predisposição para vivenciar eventos estressores de forma negativa. Os resultados sustentam a teoria de que parte da suscetibilidade para depressão e para responder com depressão aos eventos de vida estressores esteja ligada a um mesmo conjunto de genes entre as meninas púberes.

Ao transportarmos esses resultados para o campo dos transtornos de ansiedade, é lícito imaginarmos que a interação de fatores ambientais (os eventos de vida estressores) com predisposição genética para transtornos de ansiedade, modulada pelas capacidades do sujeito em lidar com estes estressores, determinada também geneticamente, resultaria no surgimento de um transtorno de ansiedade.

Para testar a relação causal entre eventos de vida estressores independentes e o aparecimento de transtornos de ansiedade ou depressão, Silberg e cols. (2001) realizaram estudo de coorte prospectiva com 184 pares de gêmeas. ${ }^{4}$ A hipótese de que transtornos de ansiedade ou depressão estão mais provavelmente associados com eventos de vida estressores negativos independentes em indivíduos com suscetibilidade genética para lidar de forma inapropriada com esses eventos foi avaliada. O estudo envolveu meninas entre 14 e 17 anos, e seus resultados demonstraram não haver efeito genético sobre os eventos de vida estressores independentes estudados, exceto na presença de doença mental parental, quando o efeito dos eventos de vida estressores independentes esteve relacionado com o surgimento de depressão, havendo uma interação estatisticamente 
significativa entre herança genética para depressão e ambiente (evento de vida estressor independente). Os autores concluem que, para que os eventos de vida estressores desempenhem um papel etiológico sobre o surgimento de sintomas de depressão ou ansiedade, deve haver ou uma predisposição genética para lidar de forma inadequada com esses eventos, ou uma vulnerabilidade aumentada resultante de efeitos ambientais de doença mental parental.

Em síntese, eventos de vida estressores podem ser entendidos como preditores ambientais de ansiedade e depressão. Fatores genéticos desempenham um papel nas diferenças de suscetibilidade individual a estes eventos.

\section{ESTRESSE E ANSIEDADE}

A hipótese de que a severidade ou presença de eventos de vida estressores são preditivos de severidade ou presença de sintomas de ansiedade ou de transtornos de ansiedade têm sido alvo de estudos recentes com adultos e adolescentes.

A relação etiológica entre a exposição a eventos de vida estressores e o surgimento de sintomas e transtornos de ansiedade em geral, apesar de plausível, tem sido pouco estudada. Pouco se sabe sobre como as mudanças na carga de estresse ao longo do tempo se relacionam com as mudanças nos sintomas prodrômicos de ansiedade e no desenvolvimento de um transtorno de ansiedade.

Sabe-se que os sintomas prodrômicos de ansiedade podem surgir anos antes do surgimento de um transtorno definido e completo, em resposta a eventos estressores, como por exemplo desavenças interpessoais entre pacientes adultos. Então, estressores desta natureza são co-responsáveis pelo surgimento de transtornos mentais a curto, médio e longo prazo, bem como podem precipitar a recorrência de quadros psiquiátricos. ${ }^{15}$

Reuter e cols. (1999) ${ }^{15}$, em uma coorte prospectiva com 303 adolescentes, de ambos os sexos, entre 12 e 13 anos, avaliados anualmente por quatro anos, testou a relação entre desavenças entre pais e filhos como evento de vida estressor, sintomas prodrômicos de ansiedade e depressão e o surgimento de transtornos de ansiedade e depressão aos 19 e 20 anos. Entre os adolescentes, a presença persistente ou crescente de desavenças com os pais foi preditiva de sintomas de ansiedade e depressão. A presença de sintomas crônicos ou crescentes de ansiedade ou depressão foi preditiva de transtornos de ansiedade ou de- pressão. As taxas de transtornos de ansiedade foram de $6,9 \%$ entre as meninas e $5,6 \%$ entre os meninos. Este estudo é uma rara demonstração da relação causal entre os eventos de vida estressores e o surgimento de sintomas de ansiedade e depressão, reforçando a associação entre estresse, sob a forma de desavenças interpessoais, e sintomas de ansiedade e depressão. Foi demonstrada uma associação positiva entre a mudança no padrão de estresse relacionada à mudança no padrão dos sintomas, ou seja, quanto maior a exposição ao estressor mais intensa a sintomatologia. Os eventos estressores estiveram indiretamente relacionados ao surgimento dos transtornos de ansiedade e depressivos. ${ }^{15}$

Os indivíduos na fase adulta com freqüência deparam-se com diferentes situações potencialmente estressoras como a criação dos filhos, o relacionamento enquanto casal, relações interpessoais, necessidade de manutenção do emprego e a própria aposentadoria. Esta, podendo ocorrer na meia-idade, pode ser vivenciada como uma perda (financeira ou das relações sociais) ${ }^{16}$. Considerando eventos de vida estressores como infidelidade, ameaça de separação e agressões físicas, Cano \& O'Leary $(2000)^{17}$ avaliaram a relação destes eventos com sintomas depressivos e ansiosos. Os indivíduos envolvidos em situações maritais humiIhantes referiam significativamente mais sintomas não específicos de depressão e ansiedade que os indivíduos controles.

Deve-se ressaltar ainda a importância de avaliar as situações estressoras e suas manifestações entre os indivíduos da terceira idade. Nesta faixa etária, eventos de vida estressores, como perdas do companheiro, dos amigos, do trabalho e a diminuição das capacidades físicas, podem desencadear sintomatologia psiquiátrica ${ }^{16}$. Beurs e cols. $(2000)^{18}$, avaliando idosos com e sem sintomas de ansiedade, não identificaram eventos de vida estressores específicos diretamente relacionados a cronicidade de sintomas ansiosos, mas observaram, no grupo estudado, que o principal evento de vida estressor na terceira idade associado com ansiedade foi a morte do parceiro. Outro estudo realizado pelos mesmos autores $(2001)^{19}$ demonstrou existir similaridade para vulnerabilidade para depressão e ansiedade, mas os eventos de vida estressores diferiam. O início da depressão estava mais relacionado à morte do companheiro ou outro familiar e o dos sintomas ansiosos com o fato do parceiro desenvolver uma doença grave. 


\section{CONSIDERAÇÕES FINAIS}

Frente a uma situação estressora, o tipo de resposta de cada indivíduo depende, não somente da magnitude e freqüência do evento de vida estressor, como também da conjunção de fatores ambientais e genéticos. Mesmo as capacidades individuais de interpretar, avaliar e elaborar estratégias de enfrentamento parecem ser geneticamente influenciadas.

A resposta de enfrentamento ao evento estressor, selecionada a partir dos componentes cognitivo, comportamental e fisiológico, caso consiga eliminar ou solucionar a situação estressora provocará uma diminuição da cascata fisiológica ativada. Se a resposta ao estresse gerar ativação fisiológica freqüente e duradoura ou intensa, pode precipitar um esgotamento dos recursos do sujeito com o aparecimento de transtornos psicofisiológicos diversos, podendo predispor ao aparecimento de transtornos de ansiedade entre outros transtornos mentais. O desenvolvimento de um transtorno está diretamente relacionado à freqüência e duração de respostas de ativação provocadas por situações que o sujeito avalia como estressoras para si.

Diversos estudos avaliaram a relação entre a ocorrência de eventos de vida estressores e o surgimento de sintomas depressivos. No entanto, poucos dados são encontrados na literatura em relação a sintomas ansiosos frente a estes eventos. Os autores sugerem que mais estudos de enfoque etiológico sejam realizados, avaliando a relação causal entre a exposição a diferentes eventos de vida estressores e o surgimento de sintomas de ansiedade, bem como de transtornos ansiosos. O reconhecimento desta relação causal terá implicações práticas tão relevantes como a prevenção de transtornos ansiosos e o estabelecimento de estratégias de tratamento.

\section{REFERÊNCIAS BIBLIOGRÁFICAS}

1. Houaiss A, Villar MS, Franco FM. Diccionario da Língua Portuguesa. Rio de Janeiro, 1ª edição, 2001, p 1264.

2. Labrador FJ, Crespo M. Evalución del estrés. In: Fernandéz-Ballesteros R. Evaluación conductual hoy. Un enfoque para el cambio en psicologia clínica y de la salud. Ediciones pirámide S.A - Madrid; 1994. p. 484-529.

3. Organização Mundial de Saúde. Classificação de transtornos mentais e de comportamento da CID-10. Descrições clínicas e diretrizes diagnósticas. Porto Alegre: Artes Médicas; 1993.

4. Silberg J, Rutter M, Neale M, Eaves L. Genetic moderation of environmental risk for depression and anxiety in adolescent girls. British J Psychiatry 2001, 179: 116-121.
5. Yehuda R, Davidson J. Clinician's Manual on Posttraumatic Stress Disorder. Science Press, London; 2000.

6. Graeff FG. Ansiedade. In: Graeff FG, Brandão ML, editores. Neurobiologia das Doenças Mentais. 4 ed. São Paulo:Lemos; 1997. p: 109-144.

7. Ribeiro L, Kapczinsk F. Ansiedade In: Kapczinski F, Quevedo J, Izquierdo I, editores. Bases Biológicas dos Transtornos Psiquiátricos. Porto Alegre. Artmed editora;2000.p.133-141.

8. Katz L, Fleisher W, Kjernisted K, Milanese P. A review of the psychobiology and pharmacotherapy of posttraumatic stress disorder. Can J Psychiatry 1996; 41: 233-38.

9. Charney D, Deutch A, Krystal J, Southwick SM, Davis M. Psychobiologic mechanisms of posttraumatic stress disorder. Arch Gen Psych. 1993;50: 295-305.

10. Chrousos GP, Gold PW. The concepts of stress and stress system disorders:overview of physical and behavioral homeostasis. JAMA 1992;267:1244-1252.

11. Vanitallie TB. Stress: a risk factor for serious illness. Metabolism 2002;51(6suppl 1):40-45.

12. Brown GW, Bifulco A, Harris TO. Life events, vulnerability and onset of depression. Some Refinements. British Journal of Psychiatry 1987; 150: 30-42.

13. Kendler KS, Karkowski LM, Prescott CA. Causal relationship between stressful life events and the onset of major depression. Am J Psychiatry 1999;156: 837-841.

14. Silberg J, Pickles A, Rutter M, Hewitt J, Simonoff E, Maes $H$, Carbonneau R, Murrele L, Foley D, Eaves L. The influence of genetic factors and life stress on depression among adolescent girls. Arch Gen Psychiatry 1999; 56: 225-232.

15. Rueter MA, Scaramella L, Wallace LE, Conger RD. First onset of depressive or anxiety disorders predicted by the longitudinal course of internalizing symptoms and parentadolescent disagreements. Arch Gen Psychiatry 1999; 56: 726-732.

16. Margis R, Cordioli AV. Idade Adulta: meia idade In: Eizirik, CL; Kapczinski, F; Bassols, MAS. O Ciclo da Vida Humana: uma perspectiva psicodinâmica. ARTMED 2001

17. Cano A, O'Leary KD. Infidelity and separations precipitate major depressive episodes and symptoms of nonspecific depression and anxiety. J Consult Clin Psychol 2000; 68(5): 774-781.

18. De Beurs E, Beekman AT, Deeg DJ, Van Dyck R, Van Tilburg W. Predictors of change in anxiety symptoms of older persons: results from the Lngitudinal Aging Study Amsterdam. Psychol Med 2000; 30 (3):515-527.

19. De Beurs E, Beekman A, Geerlings S, Deeg D, Van Dyck $\mathrm{R}$, Van Tilburg $\mathrm{W}$ et al. On becoming depressed or anxious in late life: similar vulnerability factors but different effects of stressful life events. Br J Psychiatry 2001; 179:426-431.

\section{RESUMO}

Os autores apresentam uma breve revisão de literatura sobre a relação entre ansiedade, eventos estressores e estresse. São descritas as diferentes situações estressoras, a definição de evento de vida estressor e os aspectos cognitivos, comportamentais e fisiológicos da resposta frente ao estresse. A neuroanatomia e os principais neurotransmissores envolvidos na resposta fisiológica de ansiedade ao estresse são descritos. Estudos genéticos que evidenciam a relação entre os eventos de vida estressores como fator de risco para ansiedade são apresentados. A relação causal entre os eventos de vida estressores e o aparecimento de ansiedade é 
abordada a partir de estudos realizados com adultos e adolescentes.

Descritores: Estresse, ansiedade, eventos de vida estressores.

\section{ABSTRACT}

The authors present a brief review of the literature on the relationship between anxiety, stressful lifeevents and stress. Stressful events are described as well as the definition of stressful life-events and the cognitive, behavioral and physiological responses to stress. Neuroanatomy and neurotransmitters involved on the anxiety response to stress are described. Genetic studies on the relationship of stressful lifeevents as risk factor to anxiety were reviewed. From several studies with adults and adolescents a cause relationship between stressful life events and anxiety is evaluated.

Keywords: Stress, anxiety, stressful life-events.

Title: Stressfull life-events, stress and anxiety

Endereço para correspondência:

Regina Margis

Rua Marquês do Pombal, 1824/102

90540-000 - Porto Alegre - RS

E-mail: margisr@portoweb.com.br

Copyright $\odot$ Revista de Psiquiatria do Rio Grande do Sul - SPRS

Fluxograma 1: Níveis de resposta biológica ao estresse

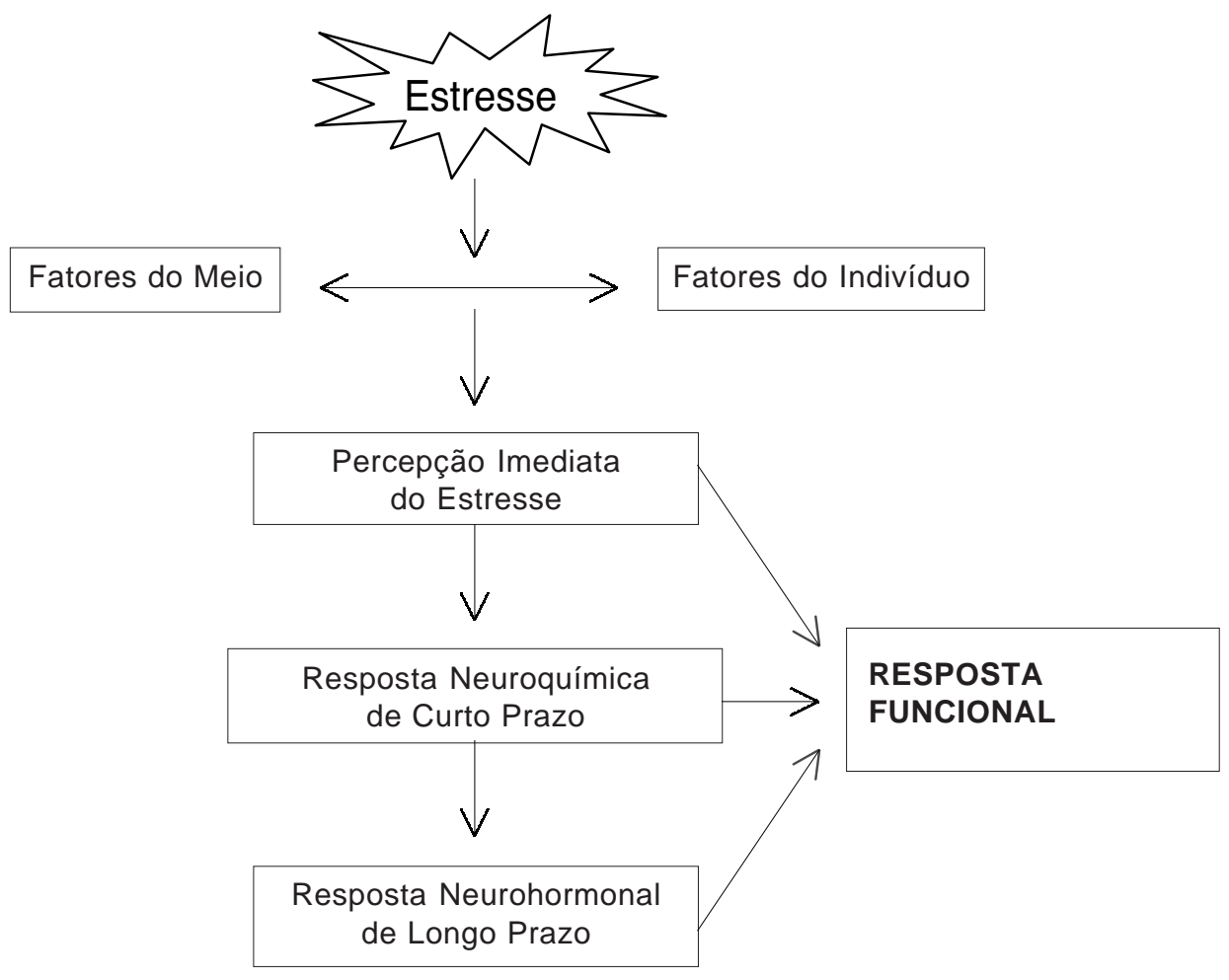


Fluxograma 2: Níveis de resposta biológica ao estresse

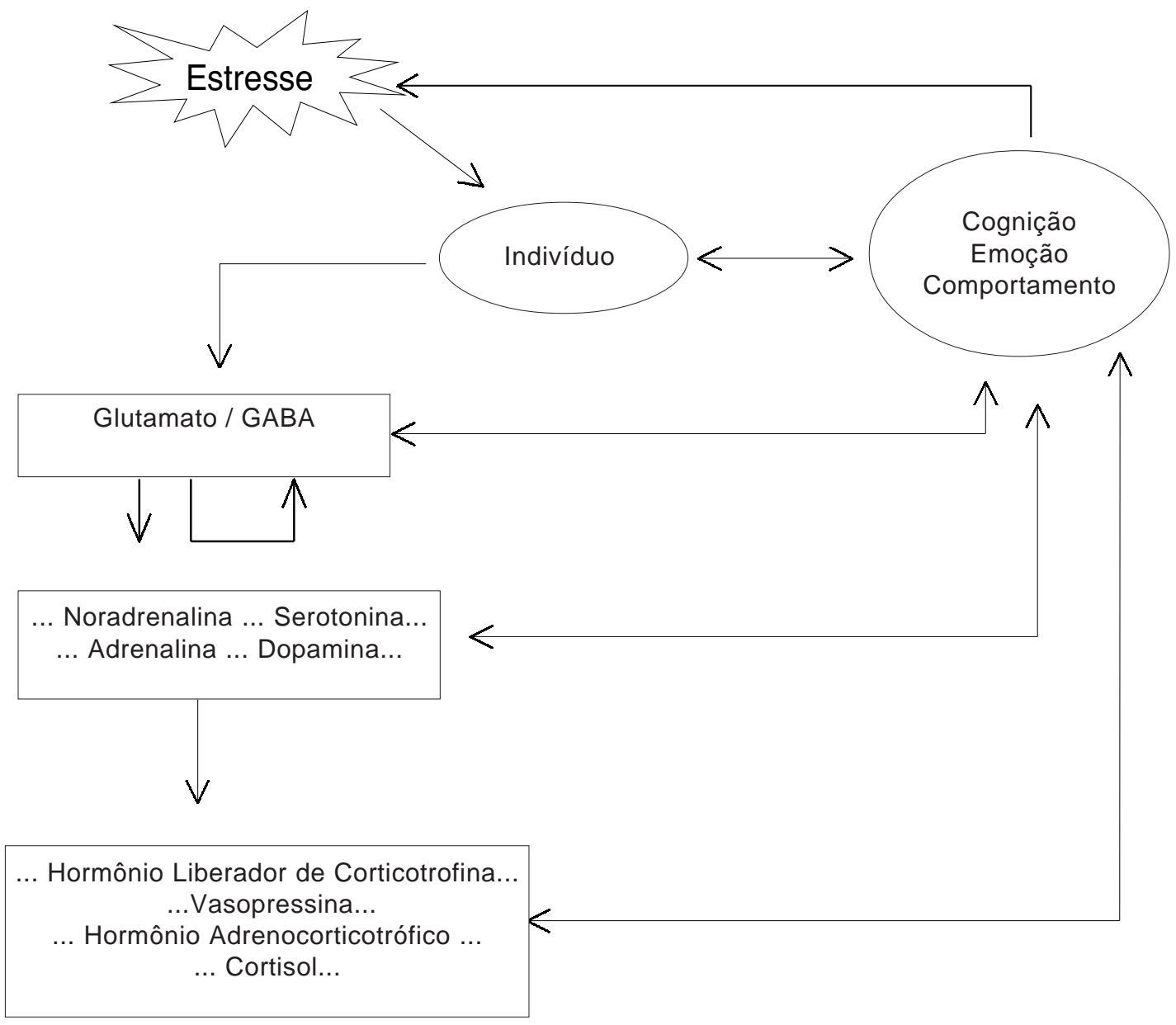

